Results There were 30 TTA prescriptions between AugustDecember 2016. 11 were handwritten and 19 were electronic. The average time to complete the process of a TTA was 20 (19-21) minutes for handwritten and 14 (12-16) minutes for a typed electronic version. The average number of items prescribed was equivocal between the groups. There were 8 enquires raised by the pharmacy team, equating to an additional $4 \mathrm{~min}$ average of extra processing time per TTA. For the first two months there were 5 enquiries raised, 4 were related to legibility. A further 3 enquiries were raised up to December and these were related to prescribing practices.

Conclusion The time taken to process TTAs has been reduced with the introduction of an electronic printed version. By learning from this cycle, we hope to continue our improvement in the discharge process by preventing delays. By using the model for improvement, small changes can help improve patient care.

\section{P-124 TO AVOID READMISSION, PATIENTS AGED 65 AND OVER ADMITTED TO HOSPITAL WITH A CARE QUALITY COMMISSION AVOIDABLE CONDITION SHOULD BE EXAMINED FOR LIFE LIMITING ILLNESSES AND CONSIDERATION GIVEN TO ADVANCE CARE PLANNING}

${ }^{1}$ Sarah Smith, ${ }^{2}$ Michael Tapley. ${ }^{1}$ Tameside Hospital, Ashton - U - Lyme, UK; ${ }^{2}$ Willow Wood Hospice, Ashton - U - Lyme, UK

\subsection{6/bmjspcare-2017-00133.123}

Background The Care Quality Commission (CQC) published the State of Care report in 2013. This highlighted the increasing number of persons aged 65 and over who had had potentially avoidable admissions to hospital with conditions such as! $>$ pneumonia and urinary infections.

Aim To investigate whether the CQC's criteria can aid admission avoidance in those with life limiting illnesses or who have multiple co-morbidities where Advance Care Planning (ACP) is appropriate.

Method Two series took place between October 2014 and August 2016. The first at Tameside Hospital reviewing deaths of those aged 65 and over. The second included those transferred to Willow Wood Hospice for end of life care.

Results

\begin{tabular}{lll}
\hline Status & $\begin{array}{l}\text { Series 1 (\%) } \\
\mathrm{n}=31\end{array}$ & $\begin{array}{l}\text { Series 2 (\%) } \\
\mathrm{n}=153\end{array}$ \\
\hline CQC avoidable admission condition & 12.9 & 9.2 \\
& $\mathrm{n}=4$ & $\mathrm{n}=14$ \\
ACP appropriate - pre-existing life limiting illness or & 22.3 & 18.3 \\
comorbidities & $\mathrm{n}=7$ & $\mathrm{n}=28$ \\
Both a CQC avoidable admission condition and ACP & 16.1 & 15.7 \\
appropriate & $\mathrm{n}=5$ & $\mathrm{n}=24$ \\
Unavoidable & 48.4 & 56.9 \\
& $\mathrm{n}=15$ & $\mathrm{n}=87$ \\
\hline
\end{tabular}

Discussion There was often a lack of agreement in cases, reflecting the complexity of admission avoidance.

Using the CQC criteria alone, between $9.2 \%$ and $12.9 \%$ of admissions could have been avoided, we disagreed and felt they were unavoidable. However, combined with those who are also appropriate for an ACP this could reduce admissions on average by $15.9 \%$.
Our aim is for a practitioner to offer ACP to inpatients at Tameside Hospital to reduce readmissions.

\section{P-125 IS AN EMERGENCY REALLY AN EMERGENCY? A FOLLOW UP STUDY OF AN EVALUATION OF URGENT ADMISSION REQUESTS TO A HOSPICE}

${ }^{1}$ Alice Harry, ${ }^{2}$ Graham Whyte, ${ }^{2}$ Emma Carduff. 'University of Glasgow, Glasgow, UK; ${ }^{2}$ Marie Curie Hospice Glasgow, Glasgow, UK

\subsection{6/bmjspcare-2017-00133.124}

Introduction Delivering 24/7 specialist palliative care is a national priority. A previous study looking at the urgent requests to the hospice, over 3 months, showed that over $3 / 4$ of appropriate admissions were admitted within 24 hours.

Aim To describe the characteristics of patients who were admitted following a request for emergency admission over a 3 month period.

Methodology This was a retrospective case note review of data for the 12 months prior to emergency admission, describing the events leading up to and the outcome of the admission.

Results Twenty-nine patients were included in the analysis. Of the 29 patients included, 34\% were from the most deprived quintile. Ninety percent of emergency referrals and 100\% of admissions had a malignant diagnosis. Forty-one percent of emergency admissions were for end-of-life care (EOLC). Sixtysix percent had a DNACPR before admission and 90\% had an electronic key information summary. Seventy-five percent had at least 1 hospital admission in the previous year but only 1 patient was admitted from hospital. Patients being admitted for EOLC or by their GP had a shorter length of admission. Seventy-two percent died during the admission and $28 \%$ were discharged home and later died at home or in the hospice. No patients died in hospital.

Conclusion The emergency admissions to the hospice over these 3 months were genuine emergencies. Most of the patients were living in deprivation, meaning they are more likely to have multiple co-morbidities and social complexities. These emergency admissions to the hospice prevented admission to hospital and furthermore any of these patients dying in hospital. Anticipatory care planning was evident but further work needs done to explore the impact of deprivation, the reasons behind the lack of emergency requests for patients with non-malignant conditions and pathways for direct hospice transfer of acute front door hospital admissions where appropriate.

\section{P-126 EFFECTIVENESS OF GABAPENTIN AND PREGABALIN FOR CANCER-INDUCED BONE PAIN: A SYSTEMATIC REVIEW}

Sophie Miller. Cicely Saunders Institute, London, UK

\subsection{6/bmjspcare-2017-00133.125}

Background Managing cancer-induced bone pain (CIBP) is challenging as background pain combined with more severe incident pain on movement makes balancing analgesia and side effects difficult. Pregabalin and gabapentin are indicated for neuropathic pain and pre-clinical studies suggest these drugs could modulate CIBP. 
Aim To examine the effectiveness of pregabalin and gabapentin for management of CIBP.

Methods A systematic review of clinical trials investigating pregabalin or gabapentin as the intervention for CIBP, with change or reduction in pain scores as outcome. Embase, Medline, Cinahl and Cochrane databases were searched from inception to March 2016. Grey literature, reference lists, conference abstracts, and hand searching of key journals were undertaken.

Results Five of 35 screened studies met inclusion criteria. Pregabalin was investigated in three RCTs, and gabapentin in one RCT and one case series, including a total of 458 patients. All studies used numerical rating scales to assess worst or average pain over 5 days- 4 weeks following pregabalin or gabapentin introduction. Two pregabalin versus placebo RCTs were methodologically strong and of these the largest and highest quality RCT showed no differences in average worst pain scores. The other RCT terminated early due to slow recruitment hence was underpowered, but indicated a small trend favouring pregabalin. A further pregabalin RCT showed significant reduction in pain scores but had methodological limitations. There was no difference in pain scores in the gabapentin versus placebo RCT, whereas the gabapentin case series reported six patients whose pain scores improved. Both gabapentin studies had design weaknesses. The drugs were well tolerated. Data heterogeneity meant meta-analysis was not possible.

Conclusion The strongest evidence to date suggests lack of effectiveness of pregabalin for CIBP. Future well conducted trials should incorporate subgroup analysis of differing primary tumour types and metastases location when evaluating these neuropathic agents in CIBP.

\section{P-127 ADOPTING AN INTEGRATIVE APPROACH WHEN TEACHING "ADVANCED COMMUNICATION"}

1,2Elizabeth Woods, ${ }^{1,2}$ Paul Paes. 'Northumbria Healthcare NHS Foundation Trust, Newcastle, UK; ${ }^{2}$ Newcastle University Medical School, UK

\subsection{6/bmjspcare-2017-00133.126}

Background Studies have shown that graduating medical students consistently feel poorly prepared to communicate in difficult clinical situations and at the end of life. ${ }^{1}$ These discussions should not be seen in isolation from other communication. We are concerned that specifically teaching advanced communication in conjunction with end of life care might result in students developing 'palliative communication skills', to be used only in specific situations rather than a holistic communication style. We aimed to show the utility of the same skills across a spectrum of communication themes in a range of settings, such as deciding whether to initiate a statin to anticipatory care planning.

Methods The project involved a new communication programme, integrating previously separate strands to deliver four half-day sessions for fifth year medical students. The students were given an "Advanced Communication Skills handbook" covering the key areas of:

- Sensitive discussions (breaking bad news)

- Decision discussions (risk and escalation)

- Challenging discussions (strong emotions and errors)

The programme used role-play and was delivered by several different disciplines to symbolically emphasise integration.
Results The students scored their ability (out of 10) to discuss the key areas listed above prior to the first session and after the final session. The results showed an increase in perceived skill level for each key area and written feedback about the sessions was very positive.

Breaking bad news: 5.3 to 7.8

Shared decision-making: 6.1 to 7.8

DNACPR discussions: 4 -to 7.4

Conclusion The feedback for these sessions, in conjunction with the increase in perceived skill level across the key areas suggests that this is an effective way of integrating communication strands and decision making in under-graduate teaching.

\section{REFERENCE}

1. Sullivan AME et al. End-of-life care in the curriculum: a national study of medical education deans. Academic Medicine 2004;79(8):760-8.

\section{P-128 USE OF A WRITTEN CARE PLAN TO ACHIEVE THE "FIVE PRIORITIES FOR CARE" AT THE END OF LIFE AT NORTHWICK PARK HOSPITAL}

Katherine Mackay, Charlotte Clare, Karen Bird, Jane Cowap, lan Rudrum. Northwick Park Hospital, London North West Healthcare NHS Trust, London, UK

\subsection{6/bmjspcare-2017-00133.127}

Background The "Five Priorities for Care" (5 PFC) at the end of life were established after the Liverpool Care Pathway (LCP) was phased out. In order to meet these priorities, London North West Healthcare created the "Last Days of Life Care Agreement" (LDLCA); a template for end of life discussions.

A 2016 audit of the use of the LDLCA within Northwick Park Hospital showed that it was not being adequately filled out. There was poor documentation surrounding end of life symptom assessment, ongoing discussions with family members and acknowledgement of spiritual needs.

As a result, the LDLCA was re-written and structured around the 5 PFC. A "symptom chart" from another trust ${ }^{1}$ was introduced to record the severity of end of life symptoms. The new LDLCA was trialled on four wards and its impact on end of life care was re-audited.

Methods All patients on the new LDLCA were reviewed over an 11 week period from $9^{\text {th }}$ August $2016(n=18)$. Notes were assessed for evidence of the 5 PFC being achieved and frequency of end of life symptom assessment.

Results All 5 PFC were achieved in 100\% of patients. An end of life discussion was had with $100 \%$ of relatives prior to commencing the LDLCA. Ongoing discussions with families were documented in 61\% cases (previously 16\%). Assessment of spiritual needs was done in 67\% (previously 27\%). There was evidence of symptom assessment in $100 \%$ of patients however these were only recorded on a four-hourly basis in $56 \%$.

Conclusions A written care agreement can create a care plan focussed on the $5 \mathrm{PFC}$. Creating this from a discussion produces individualised care and reduces the risk of "tick-box" care seen with the LCP. A specialised "symptom chart" can aid assessment of end-of-life symptoms and help achieve the care plan.

\section{REFERENCE}

1. Mason LD. Brighton \& Sussex University Hospitals NHS Trust. 